OPEN ACCESS

Edited by:

Stavros Plessas,

Democritus University of Thrace,

Greece

Reviewed by: Huaxi Yi,

Ocean University of China, China

Angelica Thomaz Vieira,

Federal University of Minas Gerais,

Brazi

*Correspondence:

Antonio Bevilacqua

antonio.bevilacqua@unifg.it

Maria Rosaria Corbo

mariarosaria.corbo@unifg.it

Specialty section:

This article was submitted to

Food Microbiology,

a section of the journal

Frontiers in Microbiology

Received: 28 May 2020 Accepted: 17 August 2020 Published: 16 October 2020

Citation:

Campaniello D, Bevilacqua $A$, Speranza $B$, Sinigaglia $M$ and

Corbo MR (2020) Alginateand Gelatin-Coated Apple Pieces as Carriers for Bifidobacterium animalis

subsp. lactis DSM 10140

Front. Microbiol. 11:566596. do: $10.3389 /$ fmicb.2020.566596

\section{Alginate- and Gelatin-Coated Apple Pieces as Carriers for Bifidobacterium animalis subsp. lactis DSM 10140}

\author{
Daniela Campaniello, Antonio Bevilacqua*, Barbara Speranza, Milena Sinigaglia and \\ Maria Rosaria Corbo*
}

Department of the Science of Agriculture, Food and Environment, University of Foggia, Foggia, Italy

Fruit and vegetables are considered good natural supports for microorganisms; however, probiotics could cause negative changes on some organoleptic and sensory traits. Thus, the main topic of this paper was the design of coated apple chips as carriers for probiotics with a high level of sensory traits. The research was divided into two steps. First, four functional strains (Limosilactobacillus reuteri DSM 20016, Bifidobacterium animalis subsp. lactis DSM 10140, and Lactiplantibacillus plantarum c16 and c19) were immobilized on apple pieces through dipping of fruit chips in probiotic suspensions for different contact times (from 15 to $30 \mathrm{~min}$ ) and stored at $4^{\circ} \mathrm{C}$ for 12 days. Periodically, the viable count was assessed. As a result of this step, a contact time of 15 min was chosen because it assured an optimal deposition of microorganisms. In the second step, apple pieces inoculated with $B$. animalis subsp. lactis DSM 10140 were coated with alginate and gelatin and stored at 4 and $8^{\circ} \mathrm{C}$ for 10 days; $\mathrm{pH}$, microbiological counts, color (browning index), and sensory scores were evaluated. Bifidobacterium animalis DSM 10140 exerted a negative effect on apple chips and cause a significant browning; however, the use of coating counteracted this phenomenon. In fact, coated chips showed higher sensory scores and lower browning index. In addition, gelatin showed better performances in terms of probiotic viability, because at $8^{\circ} \mathrm{C}$, a significant viability loss of $B$. animalis DSM $10140(1.2 \mathrm{log} \mathrm{cfu} / \mathrm{g})$ was found on alginate-coated chips. Gelatin-coated apple pieces with B. animalis subsp. lactis DSM 10140 could be an attractive functional food for a wide audience, although further investigations are required on in vivo effects of this product after consumption.

Keywords: apples, edible coatings, probiotics, alginate, carriers, gelatin

\section{INTRODUCTION}

Cell immobilization is the physical confinement of intact cells to a well-defined region of space; a requisite of immobilization is that cells retain their biological activity (Karel et al., 1985). This technology was successfully applied on food-grade microorganisms for technological purposes; for example, Moreno-García et al. (2018) reported that it is possible to confine intact and active yeasts to a specific region, increasing cell density, enhancing some metabolic pathways, improving the 
control and stability of the strain, providing cell protection against shear forces, and enabling cell recovery/reutilization.

Microorganisms (yeasts or bacteria) could be immobilized on various gels (polyacrylamide, alginate, pectinates, carrageenan, etc.) either of industrial origin (porous fibers, polyurethane foams, membranes, etc.) or natural materials (Poreda et al., 2013).

Fruit and vegetables are considered good natural supports for microorganisms, because of some positive properties, like the presence of ridges on the plant surface microarchitecture, the content in prebiotics, which protect probiotic microorganisms from the acidic environment of the stomach, and other nutrients (carbohydrates, vitamins etc.). Fruits are composed of non-digestible carbohydrates, which are the base for cell immobilization; due to their cellulose content, some fruits, such as apples and pears, may exert a protective effect on the probiotic microorganisms during passage through the intestinal tract allowing these microorganisms (such as Lacticaseibacillus casei) to reach the colon and benefit the host (Kourkoutas et al., 2006).

Fruit pieces are natural carriers considered very interesting to enhance the aroma characteristics of many products thanks to their abundance, low cost, and food-grade composition (Kandylis et al., 2012). Apple and quince pieces proved to be appropriate carriers for immobilization of $L$. casei cells (Kourkoutas et al., 2006), Lactiplantibacillus plantarum (Speranza et al., 2018), and Saccharomyces cerevisiae (Altieri et al., 2019).

On the other hand, modern consumers, increasingly attentive toward their health, have shown a growing interest in novel foods that are capable of preventing and/or curing illness (Mitropoulou et al., 2013). These healthy foods or "functional foods" exert beneficial effects, in addition to the traditional nutritional functions: they contain bioactive compounds, like dietary fiber, oligosaccharides, and active "friendly" bacteria as probiotics; some researchers reported that probiotic foods represent ca. $60-70 \%$ of the functional foods (Thakur et al., 2015). For a long time, probiotics foods have been a part of dairy products; however, some leading factors, like the increasing number of lactose-intolerant individuals or with allergy to milk proteins, as well as the emerging trend of vegetarianism, contributed to a renewed interest on not-dairy probiotic foods (mainly fruits and vegetables) (Valero-Cases et al., 2020).

The main aim of this paper was to design a carrier for functional microorganisms with apple pieces. This study was divided into different steps as follows: (i) adsorption of four functional strains (Limosilactobacillus reuteri DSM 20016, Bifidobacterium animalis subsp. lactis DSM 10140, and two L. plantarum strains) on apple pieces, focusing on the evaluation of strain survival and on the duration of the contact probiotic solution/fruits to design performing food-like systems; (ii) evaluation of the performances of B. animalis subsp. lactis DSM 10140 immobilized on apple pieces surrounded by two different coatings (alginate and gelatin) and stored at 4 and $8^{\circ} \mathrm{C}$ for 10 days, with a focus on the viability of the strain, sensory scores, and color of apple chips.

\section{MATERIALS AND METHODS}

\section{Strains}

Limosilactobacillus reuteri DSM 20016 (Deutsche Sammlung von Mikroorganismen), Bifidobacterium animalis subsp. lactis DSM 10140, and Lactiplantibacillus plantarum c16 and c19 (Bevilacqua et al., 2010) were at $-20^{\circ} \mathrm{C}$ in MRS broth (Oxoid, Milan, Italy) $+33 \%$ sterile glycerol (J.T. Baker, Milan, Italy).

Before each experiment, the strains were cultured in MRS broth (L. reuteri DSM 20016 and L. plantarum c16 and c19 at $30^{\circ} \mathrm{C}$ for $48 \mathrm{~h}$ and B. animalis subsp. lactis DSM 10140 at $37^{\circ} \mathrm{C}$ for $48 \mathrm{~h}$ under anaerobic conditions). Then, the cultures were centrifuged at $1200 \times g$ for $10 \mathrm{~min}$ and the pellet was washed with sterile distilled water.

\section{Immobilization on Apple Pieces}

Dipping solutions for the different treatments are reported in Table 1. The adsorption of the strains on apple pieces was achieved by a modified version of the method reported by Kopsahelis et al. (2007); peeled apple pieces of Granny Smith variety, approximately $2 \times 2 \mathrm{~cm}(4.5 \mathrm{~g})$, were used as support materials. Before immobilization, they were dipped into antibrowning $(\mathrm{AB})$ solution, in order to prevent browning. For each strain, apple pieces (20-30 pieces) were introduced in $250 \mathrm{ml}$ of cell suspensions $\left(10^{7}-10^{8} \mathrm{cfu} / \mathrm{ml}\right)$ for different times $(15$, 20, 25, and $30 \mathrm{~min}$ ) (Altieri et al., 2019). After the dipping, the pieces were removed from the solution and stored into sterile containers at $4^{\circ} \mathrm{C}$ for 12 days. Apple pieces without microorganisms represented the control.

\section{Evaluation of the Performances of B. animalis Subsp. lactis DSM 10140 Immobilized on the Apple Pieces With Alginate and Gelatin Coating}

For gelatin coating, apple pieces were treated as follows: (i) dipping in $\mathrm{AB}$ solution, (ii) dipping in probiotic suspension for $15 \mathrm{~min}$, (iii) dipping in gelatin solution, (iv) air drying for $10 \mathrm{~min}$, and (v) storage at 4 and $8^{\circ} \mathrm{C}$.

For alginate coating, samples were prepared by a modified version reported by Speranza et al. (2018). Apple pieces were first dipped into $\mathrm{AB}$ solution and then in alginate solution containing the probiotic $\left(10^{8} \mathrm{cfu} / \mathrm{ml}\right)$ for $15 \mathrm{~min}$; then, a dipping in $\mathrm{CaCl}_{2}$ for $5 \mathrm{~min}$ was done, followed by air drying for $10 \mathrm{~min}$ and storage in sterile boxes at 4 and $8^{\circ} \mathrm{C}$.

Apple pieces with alginate or gelatin coating but without bifidobacteria were used as controls.

\section{Microbiological Analyses}

Twenty five grams of apples were diluted into a sterile saline solution $(0.9 \% \mathrm{NaCl})$ to obtain a 10 -fold dilution and homogenized through a laboratory blender. Then, the homogenates were serially diluted, and the viable count was evaluated through the spread plate method on MRS agar; Petri dishes were incubated under anaerobic conditions (Anaerogen kit, Oxoid) at $30^{\circ} \mathrm{C}$ for L. reuteri DSM 20016 and L. plantarum c16 and $\mathrm{c} 19$ and $37^{\circ} \mathrm{C}$ for Bifidobacterium strain for $48 \mathrm{~h}$. 
TABLE 1 | Dipping solutions.

Dipping solutions

Anti-browning (AB)

Gelatin

Alginate $(A)$

$\mathrm{CaCl}_{2}$ (hardening)
Composition

Citrate $0.2 \%(\mathrm{w} / \mathrm{v})$, ascorbate $1 \%(\mathrm{w} / \mathrm{v})$, sterile water

Sucrose $0.5 \%(\mathrm{w} / \mathrm{v})$, corn starch $0.08 \%(\mathrm{w} / \mathrm{v})$, lemon juice $0.05 \%(\mathrm{v} / \mathrm{v})$, distilled water Alginate powder $2 \%(\mathrm{w} / \mathrm{v})$, melted into sterile distilled water at $80^{\circ} \mathrm{C}$

$\mathrm{CaCl}_{2}$ 0.5\%(w/v)

\section{Colorimetric Analysis}

Color was measured using a tristimulus colorimeter CR300 Minolta Chromameter-2 Reflectance (Minolta, Japan); the measurement was performed on 10 different pieces of apple per sample. The calibration of colorimeter was done on a standard white plate $\left(\mathrm{L}^{*}=97.03, \mathrm{a}^{*}=+0.01\right.$, and $\left.\mathrm{b}^{*}=+1.63\right)$. Data were expressed according to CIELAB scale: L (luminescence), a (red/green coordinate), and b (yellow/blue coordinate).

\section{pH}

$\mathrm{pH}$ was measured on apple homogenate (10 g of apple in $90 \mathrm{ml}$ of saline solution) by a $\mathrm{pH}$ meter Crison, model micro pH 2001 (Crison, Barcelona, Spain); the calibration of the $\mathrm{pH}$ meter was done with standard buffer solutions at $\mathrm{pH}$ 2.0, 4.0, and 7.0.

\section{Sensory Assessment}

The samples were evaluated to assess the sensory scores in terms of texture, odor, and overall acceptability. The panel consisted of 10 panelists, students, and researchers of the Department of the Science of Agriculture, Food and Environment. The samples were coded by a letter and presented individually in a random order to each panelist. The score was assigned using a scale ranging from 0 to 5 (where $0=$ very poor, $2=$ acceptability limit, $5=$ excellent).

\section{Statistics}

The experiments were performed on two independent batches and repeated twice for each batch. The results for the viable count on the second step were also modeled as decrease/increase compared to the inoculation $(\Delta C)$, while the parameter " $a$ " (green-red) was modeled as $\Delta a$ (increase of red tone or browning index).

The data on the microbiological counts, $\mathrm{pH}$, and color were analyzed by means of one-way or multi-factorial analysis of variance (ANOVA) and Tukey's test as the post hoc comparison test $(P<0.05)$, while sensory scores were treated through Kruskal-Wallis test $(P<0.05)$. Statistic was done through the software Statistica for Windows ver. 12.0 (Statsoft, Tulsa, OK).

\section{RESULTS}

The results for the first step (effect of different dipping times) are shown in Table 2. Immediately after the immobilization, the viable count of all strains on apple pieces was $>7 \log \mathrm{cfu} / \mathrm{g}$ for all contact times (15-30 $\mathrm{min})$; it did not experience a significant decrease throughout time $(P>0.05)$.

For the second step, this research focused only on Bifidobacterium animalis subsp. lactis DSM 10140 due to its interesting functional properties. Therefore, the viability of $B$. animalis subsp. lactis 10140 on apple pieces coated with sodium alginate and gelatin and stored at 4 and $8^{\circ} \mathrm{C}$ for 10 days was evaluated.

The contact time was set to $15 \mathrm{~min}$. $\mathrm{pH}$ and color measurements and sensory assessment were also performed.

The results obtained with $B$. animalis DSM 10140 were modeled as $\triangle C$ (change in the viable count) and analyzed through MANOVA, to point out the effects of time and coating (kind of sample and storage temperature). Coating and time affected $\Delta C$ as individual terms as well as in interaction with temperature, although coating was the most significant factor (Table 3).

The evaluation of the standardized effects does not provide information on "how much" an independent factor (or categorical predictor) affects the dependent variable. A quantitative estimation is possible through the decomposition of the statistical hypotheses. The decomposition for $\Delta C$ is shown in Figure 1; positive values of $\Delta C$ indicate an increase of probiotic count, while negative values mean a reduction of $B$. animalis DSM 10140 count throughout time. Generally, gelatin coating did not exert a negative effect on the viability of $B$. animalis DSM 10140; on the other hand, alginate coating caused a significant decrease of probiotic count at $8^{\circ} \mathrm{C}$ after 10 days of storage $(\Delta C,-1.2 \log \mathrm{cfu} / \mathrm{g})$.

Table 4 shows the standardized effects for the browning index $(\Delta a)$; time, temperature, and coating were statistically significant both as individual and interactive terms. In particular, time played a major role $(F=43.14)$, followed by coating $(F=34.90)$ and finally by temperature $(F=4.64)$. As expected, browning index increased throughout time (Figure 2); the decomposition of the statistical hypothesis for coating pointed out the different effects of probiotic and coating on browning index. The probiotic caused a significant increase of $\Delta a$; however, coating counteracted this effect (Figure 3). Finally, the worst effect on color (highest increase of $\Delta a$ ) was found in alginatecoated pieces, stored at $8^{\circ} \mathrm{C}$ (Figure 4).

The $\mathrm{pH}$ of apples was 2.8-2.9; alginate and gelatin coating, as well as the inoculation of microorganisms, did not affect it, probably due to the low temperature. $\mathrm{pH}$, in fact, was at 2.68-2.76 throughout storage (data not shown).

Sensory scores were also assessed; Figure 5 shows the overall quality after 3 and 10 days of storage at $4^{\circ} \mathrm{C}$. As expected, scores decreased throughout time $(P=0.02)$ mainly in apple without coating (apple) and in apple chips with B. animalis DSM 10140 and without coating (10140). Some panelists regarded as inacceptable the sample containing only the probiotic after 10 days with a score of 1 . 
TABLE 2 | Microbial cell load (log cfu/g) of Limosilactobacillus reuteri DSM 20016, Bifidobacterium animalis subsp. lactis DSM 10140, and Lactiplantibacillus plantarum c16 and c19 immobilized for 15, 20, 25, and 30 min on apple pieces and stored at $4^{\circ} \mathrm{C}$ for 12 days.

\begin{tabular}{|c|c|c|c|c|c|}
\hline \multirow[t]{2}{*}{ Microorganisms } & \multirow[t]{2}{*}{ Days } & \multicolumn{4}{|c|}{ Log cfu/g } \\
\hline & & 15 & 20 & 25 & 30 \\
\hline \multirow[t]{5}{*}{ L. reuteri DSM 20016} & 0 & $7.61 \pm 0.02$ & $7.60 \pm 0.08$ & $7.60 \pm 0.07$ & $7.38 \pm 0.02$ \\
\hline & 2 & $7.70 \pm 0.05$ & $8.00 \pm 0.06$ & $8.08 \pm 0.12$ & $8.08 \pm 0.12$ \\
\hline & 7 & $7.56 \pm 0.50$ & $7.26 \pm 0.01$ & $7.25 \pm 0.07$ & $7.64 \pm 0.54$ \\
\hline & 12 & $7.72 \pm 0.02$ & $7.20 \pm 0.01$ & $7.98 \pm 0.08$ & $7.96 \pm 0.24$ \\
\hline & & $\mathrm{ns}^{\star}$ & ns & ns & ns \\
\hline \multirow[t]{5}{*}{ Bifidobacterium animalis subsp. lactis DSM 10140} & 0 & $8.07 \pm 0.06$ & $8.20 \pm 0.14$ & $8.40 \pm 0.00$ & $8.19 \pm 0.10$ \\
\hline & 2 & $7.96 \pm 0.08$ & $7.54 \pm 0.61$ & $7.61 \pm 0.20$ & $7.12 \pm 0.16$ \\
\hline & 7 & $8.33 \pm 0.03$ & $8.30 \pm 0.01$ & $8.18 \pm 0.23$ & $8.14 \pm 0.06$ \\
\hline & 12 & $8.33 \pm 0.03$ & $8.30 \pm 0.01$ & $8.00 \pm 0.00$ & $8.06 \pm 0.08$ \\
\hline & & Ns & ns & ns & ns \\
\hline \multirow[t]{5}{*}{ L. plantarum c16 } & 0 & $7.98 \pm 0.31$ & $8.12 \pm 0.25$ & $8.00 \pm 0.23$ & $7.88 \pm 0.17$ \\
\hline & 2 & $8.36 \pm 0.34$ & $8.08 \pm 0.16$ & $8.33 \pm 0.04$ & $8.59 \pm 0.01$ \\
\hline & 7 & $8.35 \pm 0.06$ & $8.32 \pm 0.03$ & $8.32 \pm 0.03$ & $8.27 \pm 0.05$ \\
\hline & 12 & $8.16 \pm 0.02$ & $8.22 \pm 0.03$ & $8.26 \pm 0.00$ & $8.33 \pm 0.04$ \\
\hline & & Ns & $\mathrm{ns}$ & $\mathrm{ns}$ & ns \\
\hline \multirow[t]{5}{*}{ L. plantarum c16 } & 0 & $8.45 \pm 0.42$ & $8.43 \pm 0.15$ & $8.42 \pm 0.07$ & $8.33 \pm 0.09$ \\
\hline & 2 & $8.12 \pm 0.17$ & $8.26 \pm 0.18$ & $8.34 \pm 0.08$ & $8.43 \pm 0.38$ \\
\hline & 7 & $8.36 \pm 0.08$ & $8.35 \pm 0.01$ & $8.38 \pm 0.01$ & $8.43 \pm 0.04$ \\
\hline & 12 & $8.10 \pm 0.01$ & $8.20 \pm 0.04$ & $8.33 \pm 0.04$ & $8.30 \pm 0.01$ \\
\hline & & Ns & ns & ns & ns \\
\hline
\end{tabular}

Mean \pm standard deviation. *ns, the data for each contact time were analyzed by one-way ANOVA and Tukey's test and the differences were not significant (P > 0.05).

TABLE 3 | Statistical effects of coating, storage time, and temperature on $\Delta C$ value (increase/decrease of viable count) for Bifidobacterium animalis subsp. lactis DSM 10140. Multi-factorial ANOVA and Tukey's test $(P<0.05)$.

\begin{tabular}{lccc}
\hline Variables & SS & df & $\boldsymbol{F}$ \\
\hline Time & 0.44 & 4 & 3.66 \\
Temperature & $-^{\star}$ & - & - \\
Coating & 0.78 & 2 & 12.86 \\
Time*temperature & - & - & - \\
Time*coating & - & - & - \\
Temperature*coating & - & - & - \\
Time*temperature $^{*}$ coating & 0.83 & 8 & 3.42
\end{tabular}

SS, sum of squares. df, degrees of freedom; F, Fisher test. *Not significant.

The use of coating (alginate and gelatin) could counteract this phenomenon; in fact, the samples with B. animalis DSM 10140 and coating retained a higher sensory score after 10 days.

\section{DISCUSSION}

Although fermented dairy products are the most common foods through which humans take probiotics, many non-dairy or non-traditional probiotic products are now marketed in many countries (Ranadheera et al., 2017). Fruit and vegetables are rich in nutrients and, when combined with probiotics, can positively affect human health; therefore, using fruits as carriers for probiotics is highly advantageous and is widely accepted by consumers (Martins et al., 2013).

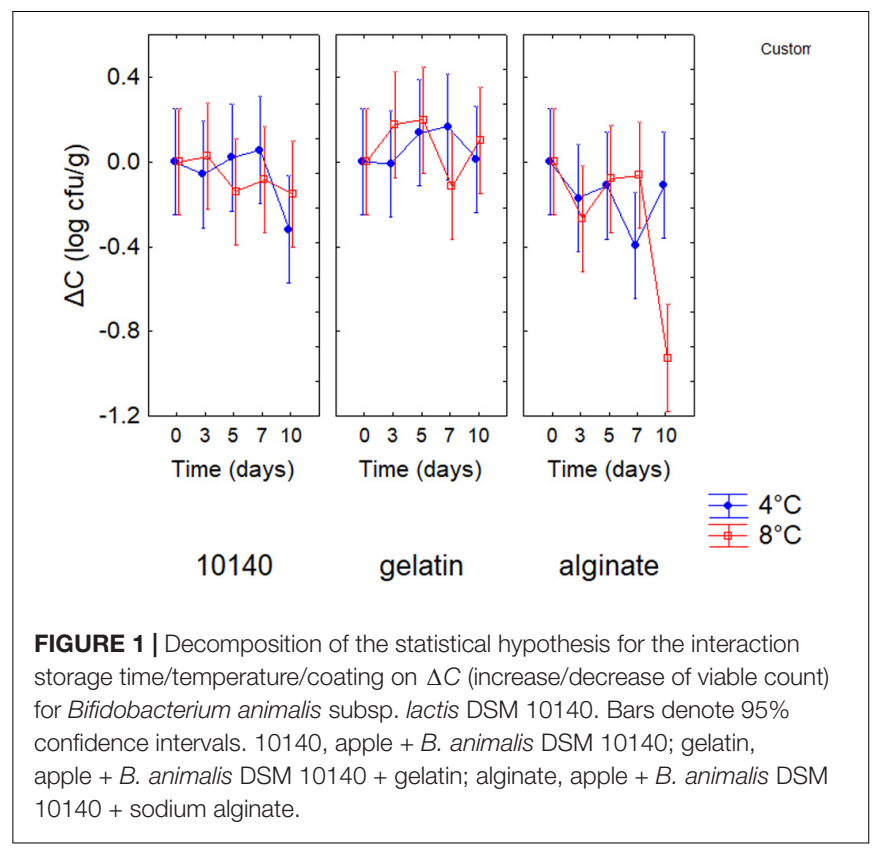

Moreover, fruits are good substrates for probiotics since they have nutrients and morphological structures favoring microbial growth (Martins et al., 2013). In addition, compared to dairy products, fruits are free of cholesterol, lactose, and allergens, and this is another reason for the emerging trend toward the consumption of non-dairy foods. 
TABLE 4 | Statistical effects of coating, storage time and temperature on $\Delta$ a value (browning index) of Bifidobacterium animalis subsp. lactis 10140. Multi-factorial ANOVA and Tukey's test $(P<0.05)$.

\begin{tabular}{lccc}
\hline Variables & SS & df & $\boldsymbol{F}$ \\
\hline Time & 125.08 & 3 & 43.14 \\
Temperature & 4.49 & 1 & 4.64 \\
Coating & 101.20 & 3 & 34.90 \\
Time*temperature & 32.35 & 3 & 11.16 \\
Time* $^{\star}$ coating & 53.80 & 9 & 6.19 \\
Temperature*coating $_{\text {Time*temperature*coating }}$ & 16.71 & 3 & 5.76 \\
\hline
\end{tabular}

SS, sum of squares. df, degrees of freedom; F, Fisher test.

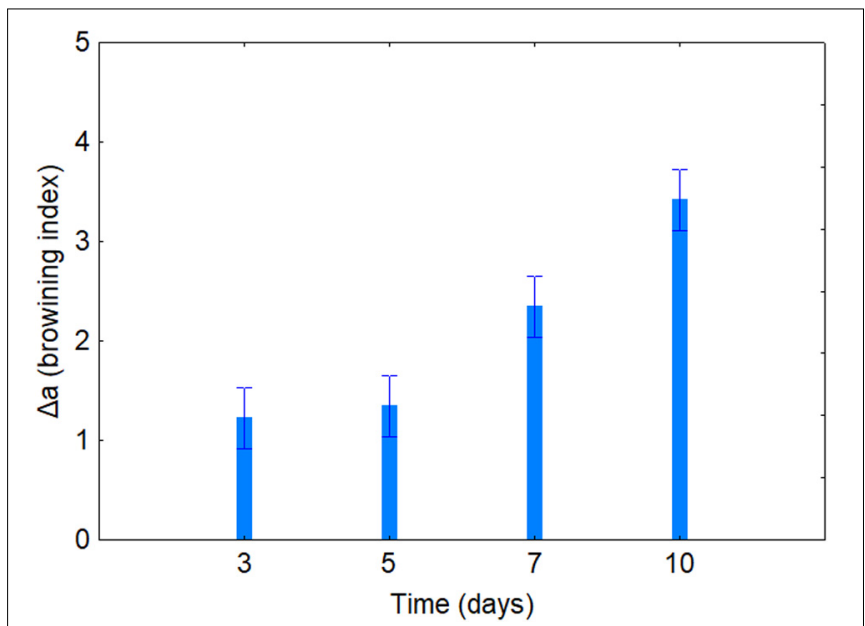

FIGURE 2 | Decomposition of the statistical hypothesis for the individual effects of time on $\Delta a$ value (browning index). Bars denote $95 \%$ confidence intervals.

Some authors proposed fruit chips as carriers for lactobacilli and yeasts (among others, Speranza et al., 2018; Altieri et al., 2019), but few evidences can be found on bifidobacteria (Rahmdel et al., 2019). Therefore, considering the potential applications of these types of products in the food industry, the feasibility of developing a protocol for the production of apple pieces as probiotic carriers with two different coatings was investigated.

The first goal of this research was to study the effect of contact time on strain adsorption on apple pieces. The immobilization of probiotics on fruit pieces is generally the result of a natural deposition of microorganisms on the surface of fruit and vegetable chips (Kourkoutas et al., 2005, 2006; Gallo et al., 2014; Russo et al., 2014, 2015; Speranza et al., 2018). The yield of strain deposition could be affected by several variables, like the shape of piece, the duration of dipping in probiotic solution, the use of static conditions or agitation, etc. Focusing on the duration of the treatment, Russo et al. $(2014,2015)$ used a dipping time of $2 \mathrm{~min}$ for bananas and cantaloupe melon, while other authors (for example, Kourkoutas et al., 2005, 2006) used a dipping time of 15 min. Preliminary results (Campaniello, unpublished results) showed that dipping for a few minutes did not assure a high

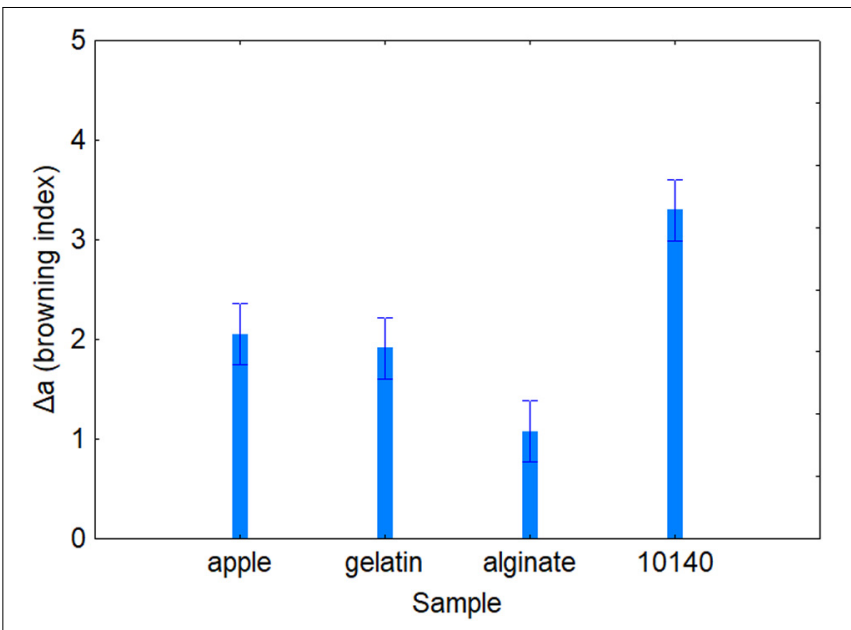

FIGURE 3 | Decomposition of the statistical hypothesis for the individual effects of coating on $\Delta$ a value (browning index). Bars denote $95 \%$ confidence intervals. Apple, control; 10140, apple + Bifidobacterium animalis subsp. lactis DSM 10140; gelatin, apple + B. animalis DSM 10140 + gelatin; alginate, apple + B. animalis DSM 10140 + sodium alginate.

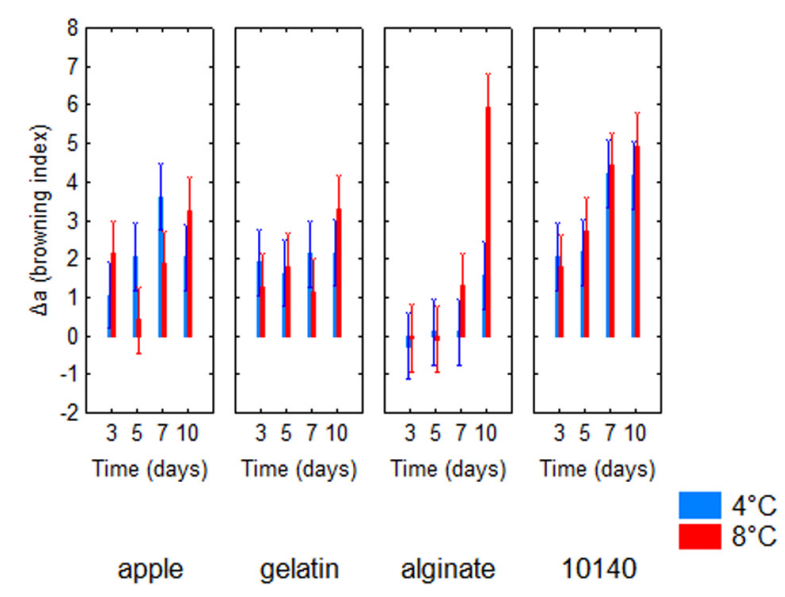

FIGURE 4 | Decomposition of the statistical hypothesis for the interaction time/temperature/coating on $\Delta$ a value (browning index). Bars denote 95\% confidence intervals. Apple, control; 10140, apple + Bifidobacterium animalis subsp. lactis DSM 10140; gelatin, apple + B. animalis DSM 10140 + gelatin; alginate, apple + B. animalis DSM 10140 + sodium alginate.

deposition of probiotics, and the viable count after the process was 5-6 log cfu/g, i.e., lower than the break point for probiotics foods. Based on these results, a dipping time of at least $15 \mathrm{~min}$ was used; however, the results showed that the level of probiotics on apple pieces was not influenced by the contact time (from 15 to $30 \mathrm{~min}$ ); thus, for the easiness of the process (a technological innovation should be feasible without affecting productivity and operational capacity), a contact time of $15 \mathrm{~min}$ (the lowest one) was regarded as acceptable for the second step of this research.

A second requisite to design probiotic food is the viability of probiotics throughout storage (technological robustness of the microbial targets); apple chips are not a "friendly" environment 


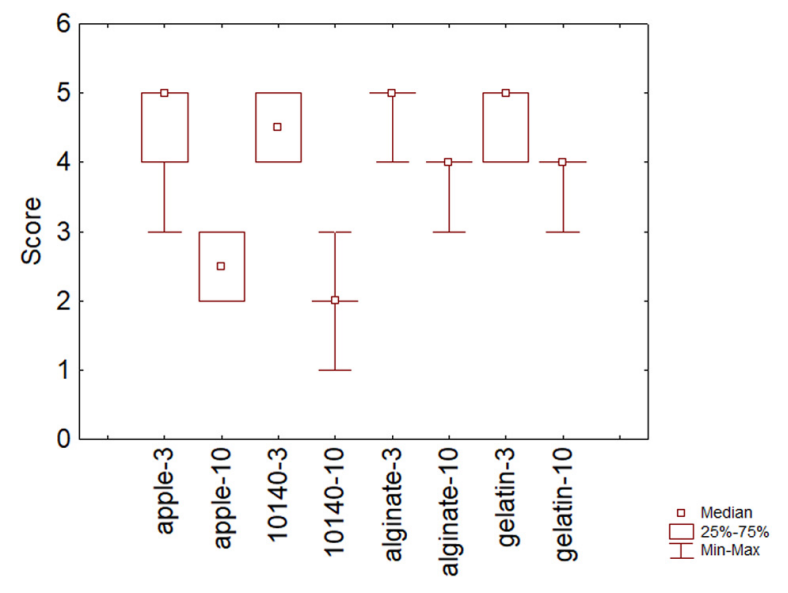

FIGURE 5 | Sensory scores on the overall acceptability after 3 and 10 days of storage at $4^{\circ} \mathrm{C}$. Apple, control; 10140, apple + Bifidobacterium animalis subsp. lactis DSM 10140; gelatin, apple + B. animalis DSM 10140 + gelatin; alginate, apple + B. animalis DSM 10140 + sodium alginate. 3 and 10, 3 and 10 days of storage.

due to the low $\mathrm{pH}$; thus, strain viability was assessed after the evaluation of the effect of contact time. Results show that all strains survived on apple pieces throughout the storage at $4^{\circ} \mathrm{C}$. These data are in line with some literature findings.

Rößle et al. (2010) loaded Lacticaseibacillus rhamnosus GG to fresh-cut apple wedges (cv. Braeburn) and found that the initial $10^{8} \mathrm{cfu} / \mathrm{g}$ inoculum remained stable throughout 10 days of storage at $2-4^{\circ} \mathrm{C}$. Rahmdel et al. (2019) used apple pieces as carriers for Bifidobacterium animalis subsp. lactis BB-12; the count was higher than the threshold (6 log cfu/g) for the whole shelf life.

For the design of coated apple chips, only B. animalis subsp. lactis DSM 10140 was used because of its technological robustness (Speranza et al., 2012; Bevilacqua et al., 2013). However, the design of a probiotic food is not a simple inoculation of microorganism and a mere evaluation of viability throughout time. In fact, it is well known that due to their active metabolism, some probiotics continue to produce lactic acid or other metabolites, thus causing negative changes on organoleptic and sensory scores (Bevilacqua et al., 2016; Racioppo et al., 2017). A negative effect of probiotics in fruit chips was found by Speranza et al. (2018) with regard to L. plantarum c19 inoculated on apple pieces.

In addition, fruits and vegetables, when processed, are more perishable than the raw material; in fact, when fruits are minimally processed, natural barriers are removed through peeling and subjected to washing, cutting, and slicing. All these treatments cause tissue injuries and damage the integrity of the fruits, and the product becomes vulnerable to contamination, enzymatic oxidations, and structural alterations.

The negative effect of probiotics was also found in this research, as suggested by the increase of the browning index (more than in the control chips) as well as by a significant decrease of the overall quality perceived by the panelist.
These effects could be the result of the pectolytic activity of Bifidobacterium spp. (Slováková et al., 2002; Flint et al., 2008) as well as of glucose/fructose metabolism. Although to some extent a partial degradation of pectin could be of interest, because bifidobacteria produce SCFA (short chain fatty acids) from some fibers, the reduction of sensory scores could be a problem.

The effects related to probiotic activity and perishable nature of minimally processed fruits can be prevented and/or counteracted through the use of artificial barriers surrounding the product as edible and coating films (Parreidt et al., 2018). In fact, edible films acting as selective barriers to moisture transfer, to oxygen uptake, to lipid oxidation, and to the loss of flavors and aromas are capable of improving the food quality (Tapia et al., 2007).

Due to its colloidal properties and ability to make strong gels or insoluble polymers, alginate is considered suitable for edible films and food coatings. Furthermore, it is classified as GRAS (generally regarded as safe) by the U.S. Food and Drug Administration (FDA), and the European Commission (EC) listed alginic acid and its salts (E400-E404) as an authorized food additive (Parreidt et al., 2018). Another possibility for edible coating is gelatin (Aguilar-Méndez et al., 2008; Gol and Ramana Rao, 2013), because of its barrier properties against oxygen and carbon dioxide (Jiang et al., 2010). Moreover, gelatin is a widespread ingredient in many formulations, and it is not perceived as a non-natural compound.

The use of alginate and gelatin coating counteracted the negative effects of both minimally processed fruits (perishable products) and probiotic (browning of apple chips) and assured the retention of sensory scores for a longer time. The performances of the two coatings were similar, although B. animalis experienced a 1-log viability loss on apple pieces with alginate stored at $8^{\circ} \mathrm{C}$. There are no evidences to give an insight into this phenomenon, and further investigations are required.

Other researchers stressed the feasibility of coated fruit pieces with probiotics. Tapia et al. (2007) proposed alginate- and gellanbased edible films for apple and papaya chips; they found that coating was able to prevent the viability loss of B. lactis BB-12 on fresh-cut apple and papaya.

Speranza et al. (2018) conducted a study on apple (and melon) pieces as carriers for L. plantarum. Alginate and chitosan were used as coatings, but the results of this study demonstrated that alginate coating provided better performances while preserving the vitality of the probiotic strain.

Mannucci et al. (2017) used gelatin as edible coating on Fuji apple to evaluate the aroma profile, and the authors observed a reduction in the acetate esters (which generally increase with maturity) that induced a delay in ripening, as an effect of the presence of gelatin coating.

The results of this paper suggest the possibility of an effective scaling up at the industrial level of apple chips with $B$. animalis subsp. lactis DSM 10140 and coated with gelatin or alginate for a better retention of sensory scores; however, there are some open questions that should be addressed. First, bifidobacteria produce SCFA from pectins; thus, it could be of interest to assess if these compounds could be produced in situ as a way to enrich apple pieces with bioactive compounds. In addition, some 
other parameters should be assessed in the light of nutritional characterization of coated apple pieces (such as vitamins, sugar, etc.) to clarify the role of probiotics on the nutritive traits.

This paper addresses the technological challenge related to the design of a new carrier for $B$. animalis subsp. lactis DSM 10140; however, a future perspective of this research could be a focus on the functional effect of this product (viability of the probiotic throughout the transit into the gut, ability to induce SCFA production in the large intestine, and effect on the gut microbiota).

\section{CONCLUSION}

This paper reports on the design of apple-based carriers for B. animalis subsp. lactis DSM 10140. The probiotic survived throughout storage at 4 and $8^{\circ} \mathrm{C}$, but it could cause a worsening of color with an increase of browning index. However, the use of a coating (alginate- or gelatin-based) counteracted this effect.

Concerning the effect of the viability, gelatin coating showed a better performance than alginate.

The formulation proposed in this research could meet the approval of a large number of consumers; in fact, due to their convenience, fresh-cut fruits are considered very attractive. Then, if these ready-to-use foods are also healthy thanks to the presence of probiotic strains, their attractiveness further increases. Moreover, the gelatin coating could make the final product suitable for different types of use (e.g., pastry).

Further studies are necessary to evaluate, for example, the role of the probiotic, carried by vegetable foods, in the intestine, as

\section{REFERENCES}

Aguilar-Méndez, M. A., San Martín-Martínez, E., Tomás, S. A., Cruz-Orea, A., and Jaime-Fonseca, M. R. (2008). Gelatine-starch films: physicochemical properties and their application in extending the post-harvest shelf life of avocado (Persea americana). J. Sci. Food Agric. 88, 185-193. doi: 10.1002/jsfa.3068

Altieri, C., Campaniello, D., Speranza, B., Sinigaglia, M., Corbo, M. R., and Bevilacqua, A. (2019). Immobilization of Saccharomyces cerevisiae on apple pieces to produce cider. Fermentation 5:74. doi: 10.3390/fermentation5030074

Bevilacqua, A., Altieri, C., Corbo, M. R., Sinigaglia, M., and Ouoba, L. I. I. (2010). Characterization of lactic acid bacteria isolated from italian bella di cerignola table olives: selection of potential multifunctional starter cultures. J. Food Sci. 75, M536-M544.

Bevilacqua, A., Campaniello, D., Corbo, M. R., Maddalena, L., and Sinigaglia, M. (2013). Suitability of Bifidobacterium spp. and Lactobacillus plantarum as probiotics intended for fruit juices containing citrus extracts. J. Food Sci. 78, 1764-1771.

Bevilacqua, A., Casanova, F. P., Petruzzi, L., Sinigaglia, M., and Corbo, M. R. (2016). Using physical approaches for the attenuation of lactic acid bacteria in an organic rice beverage. Food Microbiol. 53, 1-8. doi: 10.1016/j.fm.2015.08.005

Flint, H. J., Bayer, E. A., Rincon, M. T., Lamed, R., and White, B. A. (2008). Polysaccharide utilization by gut bacteria: potential for new insights from genomic analysis. Nat. Rev. Microbiol. 6, 121-131. doi: 10.1038/nrmicro1817

Gallo, M., Bevilacqua, A., Speranza, B., Sinigaglia, M., and Corbo, M. R. (2014). Alginate beads and apple pieces as carriers for Saccharomyces cerevisiae var. boulardii, as representative of yeast functional starter cultures. Int. J. Food Sci. Technol. 49, 2092-2100. doi: 10.1111/ijfs.12518

Gol, N. B., and Ramana Rao, T. V. (2013). Influence of zein and gelatin coatings on the postharvest quality and shelf life extension of mango (Mangifera indica L.). Fruits 69, 101-115. doi: 10.1051/fruits/2014002 well as the metabolites produced throughout storage and other nutritional traits.

\section{DATA AVAILABILITY STATEMENT}

The raw data supporting the conclusions of this article will be made available by the authors, without undue reservation, to any qualified researcher.

\section{AUTHOR CONTRIBUTIONS}

DC, MS, and MC: conceptualization. AB: software. BS and DC: investigation. $\mathrm{MS}$ and $\mathrm{MC}$ : resources. $\mathrm{AB}$ and $\mathrm{DC}$ : methodology, data curation, and writing - original draft preparation. MC: supervision and funding acquisition. DC, AB, BS, MS, and MC: writing - review and editing. All authors have read and agreed to the published version of the manuscript.

\section{FUNDING}

This research was funded by the University of Foggia, through the grant PRA 2017 (Progetti di Ricerca di Ateneo), "Use of biocompatible microbial immobilization systems for the production of food beverages" (Principal Investigator, Maria Rosaria Corbo).

Jiang, M., Liu, S., Du, X., and Wang, Y. (2010). Physical properties and internal micro-structures of films made from catfish skin gelatin and triacetin mixtures. Food Hydrocoll. 24, 105-110. doi: 10.1016/j.foodhyd.2009.08.011

Kandylis, P., Mantzari, A., Koutinas, A. A., and Kookos, I. K. (2012). Modelling of low temperature wine-making, using immobilized cells. Food Chem. 133, 1341-1348. doi: 10.1016/j.foodchem.2012.02.022

Karel, S. F., Libicki, S. B., and Robertson, C. R. (1985). The immobilization of whole cells: engineering principle. Chem. Eng. Sci. 40, 1321-1354. doi: 10.1016/00092509(85)80074-9

Kopsahelis, N., Panas, P., Kourkoutas, Y., and Koutinas, A. A. (2007). Evaluation of the thermally dried immobilized cells of Lactobacillus delbrueckii subsp. bulgaricus on apple pieces as a potent starter culture. J. Agric. Food Chem. 55, 9829-9836. doi: 10.1021/jf0719712

Kourkoutas, Y., Kanellaki, M., and Koutinas, A. A. (2006). Apple pieces as immobilization support of various microorganisms. LWT Food Sci. Technol. 39, 980-986. doi: 10.1016/j.lwt.2006.02.024

Kourkoutas, Y., Xolias, V., Kallis, M., Bezirtzoglou, E., and Kanellaki, M. (2005). Lactobacillus casei cell immobilization on fruit pieces for probiotic additive, fermented milk and lactic acid production. Process Biochem. 40, 411-416. doi: 10.1016/j.procbio.2004.01.029

Mannucci, A., Serra, A., Remorini, D., Castagna, A., Mele, M., Scartazza, A., et al. (2017). Aroma profile of Fuji apples treated with gelatin edible coating during their storage. LWT Food Sci. Techol. 85, 28-36. doi: 10.1016/j.lwt.2017.06.061

Martins, E. M. F., Ramos, A. M., Vanzela, E. S. L., Stringheta, P. C., Pinto, C. L. O., and Martins, J. M. (2013). Products of vegetable origin: a new alternative for the consumption of probiotic bacteria. Food Res. Int. 51, 764-770. doi: 10.1016/j. foodres.2013.01.047

Mitropoulou, G., Nedovic, V., Goyal, A., and Kourkoutas, Y. (2013). Immobilization technologies in probiotic food production. J. Nutr. Metab. 2013, 1-15. doi: 10.1155/2013/716861 
Moreno-García, J., García-Martínez, T., Mauricio, J. C., and Moreno, J. (2018). Yeast immobilization systems for alcoholic wine fermentations: actual trends and future perspectives. Front. Microbiol. 9:241. doi: 10.3389/fmicb.2018.00241

Parreidt, T. S., Müller, K., and Schmid, M. (2018). Alginate-based edible films and coatings for food packaging applications. Foods 7:170. doi: 10.3390/ foods7 100170

Poreda, A., Tuszyñski, T., Zdaniewicz, M., Sroka, P., and Jakubowski, M. (2013). Support materials for yeast immobilization affect the concentration of metal ions in the fermentation medium. J. Inst. Brew. 119, 164-171.

Racioppo, A., Corbo, M. R., Piccoli, C., Sinigaglia, M., Speranza, B., and Bevilacqua, A. (2017). Ultrasound attenuation of lactobacilli and bifidobacteria: effect on some technological and probiotic properties. Int. J. Food Microbiol. 21, 243-278.

Rahmdel, S., Jahed-Khaniki, G., Abdollahzadeh, S. M., Shekarforoush, S. S., and Mazloomi, S. M. (2019). Development of fresh-cut apple slices enriched with probiotic strain Bifidobacterium animalis subsp. lactis BB-12. Int. J. Probiot. Prebiot. 14, 37-44. doi: 10.37290/ijpp2641-7197.14:37-44

Ranadheera, C. S., Vidanarachchi, J. K., Rocha, R. S., Cruz, A. G., and Ajlouni, S. (2017). Probiotic delivery through fermentation: dairy vs. non-dairy beverages. Fermentation 3:67. doi: 10.3390/fermentation3040067

Rößle, C., Auty, M. A. E., Brunton, N., Gormley, R. T., and Butler, F. (2010). Evaluation of fresh-cut apple slices enriched with probiotic bacteria. Innov. Food Sci. Emerg. Technol. 11, 203-209. doi: 10.1016/j.ifset.2009.08.016

Russo, P., De Chiara, M. L. V., Vernile, A., Amodio, M. L., Arena, M. P., Capozzi, V., et al. (2014). Fresh-cut pineapple as a new carrier of probiotic lactic acid bacteria. Biomed. Res. Int. 2014:309183.

Russo, P., Peña, N., de Chiara, M. L. V., Amodio, M. L., Colelli, G., and Spano, G. (2015). Probiotic lactic acid bacteria for the production of multifunctional fresh-cut cantaloupe. Food Res. Int. 77, 762-772. doi: 10.1016/j.foodres.2015. 08.033

Slováková, L., Dušková, D., and Marounek, M. (2002). 1.1 Fermentation of pectin and glucose, and activity of pectin-degrading enzymes in the rabbit caecal bacterium Bifidobacterium pseudolongum. Lett. Appl. Microbiol. 35, 126-130. doi: 10.1046/j.1472-765x.2002.01159.x

Speranza, B., Bevilacqua, A., Sinigaglia, M., and Corbo, M. R. (2012). Shelf life definition for Italian anchovies inoculated with Lactobacillus plantarum and Bifidobacterium animalis subsp. lactis. Innov. Food Sci. Emerg. Technol. 16, 171-180. doi: 10.1016/j.ifset.2012.05.009

Speranza, B., Campaniello, D., Bevilacqua, A., Altieri, C., Sinigaglia, M., and Corbo, M. R. (2018). Viability of Lactobacillus plantarum on fresh-cut chitosan and alginate-coated apple and melon pieces. Front. Microbiol. 9:2538. doi: 10.3389/ fmicb.2018.2538

Tapia, M. S., Rojas-Grau, M. A., Rodríguez, E. J., Ramírez, J., Carmona, A., and Martin-Belloso, O. (2007). Alginate- and gellan-based edible films for probiotic coatings on fresh-cut fruits. J. Food Sci. 72, E190-E196.

Thakur, M., Satwadhar, P. N., and Deshpande, H. W. (2015). Exploiting fruits and vegetables for development of probiotic products. Trends Biosci. 8, 60396046.

Valero-Cases, E., Cerdá-Bernad, D., Pastor, J. J., and Frutos, M. J. (2020). Nondairy fermented beverages as potential carriers to ensure probiotics, prebiotics, and bioactive compounds arrival to the gut and their health benefits. Nutrients $12: 1666$.

Conflict of Interest: The authors declare that the research was conducted in the absence of any commercial or financial relationships that could be construed as a potential conflict of interest.

Copyright (c) 2020 Campaniello, Bevilacqua, Speranza, Sinigaglia and Corbo. This is an open-access article distributed under the terms of the Creative Commons Attribution License (CC BY). The use, distribution or reproduction in other forums is permitted, provided the original author(s) and the copyright owner(s) are credited and that the original publication in this journal is cited, in accordance with accepted academic practice. No use, distribution or reproduction is permitted which does not comply with these terms. 